\title{
DISTRIBUTION AND UTILISATION OF IVERMECTIN (MECTIZAN): A CHEMOTHERAPEUTIC APPROACH TO THE CONTROL OF ONCHOCER- CIASIS IN OLD OHAOZARA LGA, EBONYI STATE, EASTERN NIGERIA
}

\author{
By \\ ELOM MICHAEL OKPARA ${ }^{1}$, ALO MOSES NNAEMEKA ${ }^{2}$, \\ UGAH UCHENNA IYIOKU ${ }^{3^{*}}$ AND USANGA VICTOR UDOH ${ }^{1}$ \\ Department of Medical Laboratory Science ${ }^{1}$, Ebonyi State University, Abakaliki, and \\ Departments of Biological Sciences ${ }^{2}$, and Medical Biochemistry ${ }^{3}$, Federal University, \\ Ndufu-Alike Ikwo ( ${ }^{\star}$ Correspondence: e-mail: ugahuchennaiyioku@gmail.com)
}

\section{Abstract}

Onchocerciasis (river blindness) is a devastating, debilitating Stigmatising and incapacitating parasitic disease that is endemic in tropical and sub-tropical regions of the world, including Nigeria. Mass distribution of ivermectin (Mectizan) to the endemic parts of the world was initiated by the Onchocerciasis Control Programmes (OCPs). Absolute compliance to the regimen for up to 15 years has been reported to be effective in the control of the disease. The study was carried out in Ohaozara LGA, Onicha LGA and Ivo LGA. The three (3) LGAs made up the defunct Old Ohaozara LGA. A structured questionnaire was used to generate information on knowledge of Onchocerciasis and on the use of ivermectin by the inhabitants of the communities of the study areas. The distribution coverage of ivermectin in the study areas dating from 2010 to 2014 was ascertained with drug distribution charts obtained from Ebonyi State Health Management Board (ESHMB), Abakaliki (the point source of distribution in the state), and from the health centres in communities of old Ohaozara LGA (the service delivery points (SDPs) to inhabitants of the communities. Data was analysed using descriptive statistics. Utilization of the regimen was ascertained by determining the actual number of tablets of mectizan that was administered to the patients at the various health cenrtes (service delivery points (SDPs) in the communities. The percentage utilization of the regimen was determined by dividing the number of mectizan tablets administered to the patients at SDPs with the number of mectizan tablets supplied from state point source of distribution and multiplying by 100. A total of 347, 299 out of 1,919135 tablets of mectizan supplied to the study areas from 2010 to 2014 were actually utilized, forming an overall percentage utilization of $18.10 \%$. There was adequate supply but very poor utilization of the regimen. The poor utilization resulted from factors including locating of health centres very far from homes of some of the rural villagers, non-yearly compliance with regimen administration, poor health sensitization and education and lack of incentives or poor incentives to the village-based health workers (VBHWs). Intensification of efforts to cover the lapses in the utilization of the regimen is advocated for a more effective control of the disease.

Keywords: Distribution, Utilization, Ivermectin, Onchocerciasis, Nigeria.

\section{Introduction}

Onchocerciasis is a devastating and debilitating parasitic disease (Crump et al, 2012). It affects the aesthetic qualities of the affected parts of the body and attracts stigmatization to the patient. If untreated, its finallyassociated blindness incapacities patients especially in farming activities. River blindness is endemic in tropical and sub-tropical countries of the world, including Nigeria and Ghana in West Africa (Cox, 1993). Onchocerca volvulus, the parasite that is responsible for the disease is transmitted through the bite of a black fly that belongs to the genus Simulium. Simulium damnosum is responsible for shaping the transmission of the disease in Nigeria while other species of the insect transmit the disease in other endemic parts of the world. The flies inhabit and breed well along banks of fast-flowing welloxygenated streams and rivers surrounding vast fertile lands of most endemic communities and take their blood meal from their hosts during the cooler hours of morning or late afternoon which coincide with active farming periods of the day. Onchocerciasis 
has made farmers in endemic areas to leave their fertile lands for non-endemic areas. The debilitating nature of the disease and its associated eventual blindness have contributed negatively to the growth of national economy since less hands participate actively in farming and huge sums of money are invested on treatment of infected people.

With the perception of onchocerciasis as a public health problem, attempts have been generated towards its successful control and possible elimination. West African countries launched the Onchocerciasis Control Programme (OCP) to control the disease with the objective of eliminating it as a public health problem and mitigating its negative socio-economic impacts in the endemic regions (WHO, 2010). The first control attempt commenced with the use of Dichlorodiphenyltrichloroethane (DDT), which was eventually replaced with Temophos, a cheap and efficient organophosphorous insecticide with non-significant impacts on non-target fauna (Richards et al, 2001). Other environmental friendly insecticides were rotated against the larval and adult stages of the vector. Excision of nodules was introduced to maximally reduce the infection transmission rates and it was an effective alternative control measure in addition to the use of insecticides.

Chemotherapeutic control of onchocerciasis commenced with the introduction of Diethylcarbamazine (DEC), a drug that kills only the micro-filariae with resultant complex reactions generally termed "Mazzotti reactions". The complex adverse reactions following treatments with DEC lead to the replacement of the drug with ivermectin (Mectizan), a milder one with high larvicidal potency. The establishment of the efficacy of ivermectin generated an increased impetus in its mass distribution to widely cover endemic communities. WHO (2012) reported that about 123.3 million people worldwide required ivermectin chemotherapy in 2010. Approximately 145,000 rural communities were actively engaged in ivermectin distribution and 500 million ivermectin treatments were dispensed by about 1 million African Programme for the control of Onchocerciasis (APOC)- trained community-based drug distributors.

Ivermectin administered once yearly has been proved to be effective in the control of onchocerciasis but administration of the treatment twice per annum could be of greater efficiency, considering the possible development of drug resistance by the parasite. Onchocerca volvulus is a long-lived parasite that undergoes a five stage life cycle. The infective third stage (L3) larvae inoculated by the vectors mature within one year to adult stage, leading to the formation of subcutaneous nodules. The coiled females which are sessile remain in the formed nodules for up to 15 years (Brattig, 2004). The parasite life span is 15 years. For effective treatments to be achieved, there should be high levels of compliance in the administration of the drugs, meaning that the patients must take the mectizan tablets consistently yearly from the commencement of the regimen to the $15^{\text {th }}$ year, when the possible remaining mirofilariae must have been cleared.

River blindness is still endemic in the entire old Ohaozara LGA. Accurate records on distribution and utilization of ivermectin are needed to forestall over stocking of drugs (which leads to expiration of unused drugs) and under stocking of drugs (which leads to the availability of insufficient quantities of drugs for treatments), and ensure the availability of ivermectin tablets wherever needed by the infected patients. This will enhance the realization of the control programme. There is a dearth of information on distribution and utilization of ivermectin in communities of old Ohaozara LGA., hence the need for this study.

\section{Materials and Methods}

The study areas were the three Local Government Areas (LGAs) that made up the old Ohaozara LGA and their component communities. The three Local Government Are- 
as are Ohaozara, Onicha and Ivo. Old Ohaozara LGA is bounded at the East by Afikpo North LGA, to the West by Nkanu LGA of Enugu State (a neighbouring state to Ebonyi State), by the North, by Aninri LGA (also of Enugu State), and to the South by Afikpo South LGA. Ohaozara is situated at the southern part of Ebonyi State. Ebonyi State is characterized by tropical rainfall type of vegetation, with high temperature and high relative humidity. Rainy and dry seasons are the two prevailing seasons in the state. The rains start by April and end in November while the dry season commences by November and ends in March.

The state is surrounded by fast flowing rivers and streams with bushy and grassy banks. Ebonyi River, the river from which the state derived its name flows with its tributaries across almost all the communities and Local Government Areas of the state, including old Ohaozara LGA. Farming is the economic mainstay of the state and farm produce like cassava, rice and yams are the staple food of the people of the state.

\section{Mectizan ${ }^{\circledR}$ tablets given to patients at delivery points (SDPs) \\ Urilizarion\% $\quad \overline{\text { Mectizan tablets given from state point source of distribution }} \quad \mathrm{X} \frac{100}{1}$}

Distribution coverage of mectizan was ascertained with data obtained from Ebonyi State Health Management Board (ESHMB), Abakaliki and data from health centres in the study communities while utilization was ascertained by knowing the actual number of tablets of mectizan that was administered to patients at various health centres [service delivery point (SDPs)] in the communities.

Table 1: Annual quantities of mectizan supplied to LGAs of old Ohaozara from 2010 to 2014

\begin{tabular}{|l|l|l|l|l|l|l|}
\hline & Years & 2010 & 2011 & 2012 & 2013 & 2014 \\
\hline \multirow{3}{*}{$\underset{⿴}{\infty}$} & Ohaozara & 105,000 & 80,000 & 94,000 & 99,800 & 99,243 \\
\cline { 2 - 7 } & Onicha & 230,000 & 120,000 & 200,000 & 204,000 & 229,970 \\
\cline { 2 - 7 } & Ivo & 110,000 & 40,800 & 100,000 & 102,500 & 103,822 \\
\hline & Total & 445,000 & 240,800 & 394,000 & 406,300 & 433,035 \\
\hline
\end{tabular}

Agugwu, Oshiri Model and Akeze Model Health Centres had the highest utilizations of mectizan $^{\circledR}(36,600 ; 29,000$ \& 22,820). The health centres are respectively from Ohaoza- ra, Onicha, and Ivo LGAs of old Ohaozara. Within the five year period, a to-
Data collection was carried out using simple structured questionnaire. The questionnaire was used to generate information on knowledge of onchocercicasis and on the use of ivermectin by the inhabitants of the study areas. Also embodied in the questionnaire was information concerning signs and symptoms of onchocerciasis as well as information on possible side effects of mectizan. The distribution coverage of ivermectin (mectizan) dating from 2010 to 2014 was ascertained with drug distribution charts obtained from Ebonyi State Health Management Board (ESHMB), Abakaliki (the point source of distribution in the state), and from the health centres in communities of old Ohaozara LGA (the service delivery points, (SDPs) to inhabitants of the villages of the communities.

Statistical analysis: Data was analyzed using descriptive statistics. Percentage utilization of ivermectin (mectizan) was determined by the formula:

\section{Results}

There were variations in yearly supply of mectizan, with years 2010 and 2011 having the highest and lowest supplies respectively. Onicha LGA had the highest supply while Ohaozara LGA had the least supply over the years. Details are in tables $(1,2,3,4,5 \& 6)$. tal of 347,299 tablets of mectizan were utilized in old Ohaozara. There was a gradual drop in the utilization of the regimen from 2010 until 2014, when the least utilization of tablets was manufactured. 
Table 3 depicted Local Government Area by Local Government Area utilization of mectizan supplied to old Ohaozara from 2010 to 2014 . There was an overall poor uti- lization of the regimen (18.10\%) all over the years. The drug was highly underutilized throughout the period in Onicha LGA.

Table 2: Health center utilization of mectizan in LGAs of old Ohaozara from 2010 to 2014

\begin{tabular}{|c|c|c|c|c|c|c|c|}
\hline & Health Centers & 2010 & 2011 & 2012 & 2013 & 2014 & Total \\
\hline \multirow{6}{*}{ 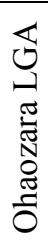 } & Obiozara $\mathrm{H} / \mathrm{C}$ & 2,600 & 2,300 & 2,900 & 2,150 & 3,030 & 13,000 \\
\hline & Agugwu H/C & 6,000 & 6,500 & 7,600 & 8,000 & 8,500 & 36,600 \\
\hline & Urban H/C, Okposi & 3,500 & 3,500 & 3,500 & 3,500 & 4,000 & 18,000 \\
\hline & Ugwulangwu $\mathrm{H} / \mathrm{C}$ & 2,160 & 2,300 & 2,000 & 3,020 & 1,370 & 11,430 \\
\hline & Ndiunuhu $\mathrm{H} / \mathrm{C}$ & 5,200 & 4,100 & 4,020 & 3,200 & 3,900 & 20,420 \\
\hline & Ojigwe $\mathrm{H} / \mathrm{C}$ & 3,600 & 3,210 & 2,600 & 2,300 & 1,342 & 13,052 \\
\hline \multirow{7}{*}{ 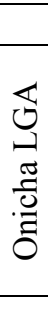 } & Sub-Total & 23,060 & 21,910 & 22,620 & 22,170 & 22,162 & 112,502 \\
\hline & Oshiri H/C & 6,200 & 5,120 & 5,000 & 4,012 & 3,220 & 23,552 \\
\hline & Isu $\mathrm{H} / \mathrm{C}$ & 5,000 & 4,200 & 3,500 & 3,200 & 3,000 & 18,900 \\
\hline & Abaomege $\mathrm{H} / \mathrm{C}$ & 2,600 & 2,160 & 3,210 & 3,100 & 2,200 & 13,270 \\
\hline & Onicha $\mathrm{H} / \mathrm{C}$ & 3,600 & 3,400 & 3,100 & 5,000 & 4,200 & 20,200 \\
\hline & Oshiri Model H/C & 7,600 & 8,400 & 5,000 & 8,200 & 8,000 & 29,000 \\
\hline & Ukawu H/C & 4,500 & 3,000 & 8,200 & 3,500 & 4200 & 20,200 \\
\hline & Sub-Total & 29,500 & 26,280 & 28,010 & 27,012 & 24,820 & 125,122 \\
\hline \multirow{8}{*}{ 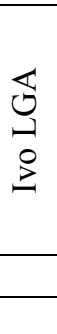 } & Ishiagu Basic H/C & 5,200 & 5,100 & 4,300 & 4,000 & 3,200 & 21,800 \\
\hline & Akeze Model H/C & 5,500 & 5,100 & 4,320 & 4,200 & 3,900 & 22,820 \\
\hline & Ihie $\mathrm{H} / \mathrm{C}$ & 4,200 & 4,101 & 3,020 & 2,230 & 2,600 & 16,151 \\
\hline & Iyioji $\mathrm{H} / \mathrm{C}$ & 4,600 & 5,202 & 4,820 & 3,202 & 2,900 & 20,724 \\
\hline & Akeze Basic H/C & 3,160 & 4,220 & 4,030 & 3,100 & 3,020 & 14,530 \\
\hline & Ekembe H/C & 2,130 & 2,220 & 3,000 & 3,200 & 3,100 & 13,650 \\
\hline & Sub-Total & 24,790 & 25,941 & 22,490 & 19,932 & 18,720 & 109,675 \\
\hline & Grand Total & 77,350 & 74,133 & 74,820 & 69,114 & 65,702 & 347,299 \\
\hline
\end{tabular}

Table 3: Local Government area utilization of mectizan supplied to old Ohaozara from 2010 to 2014

\begin{tabular}{|c|c|c|c|c|}
\hline & & Number supplied & Number utilized & Utilization $\%$ \\
\hline \multirow{4}{*}{$\sum_{=}^{\infty}$} & Ohaozara & 478,043 & 112,502 & 23.53 \\
\hline & Onicha & 983,970 & 125,122 & 12.72 \\
\hline & Ivo & 457,122 & 109,675 & 23.99 \\
\hline & Total & $1,919,135$ & 347,299 & 18.10 \\
\hline
\end{tabular}

The Health Centers in Ohaozara utilized $23.53 \%(112,502 / 478,043)$ of supplied mectizan tablets. The Ugwulangwu Health Centre highly underutilized supplied drugs, but
Agugwu Health Centre, Uburu, made highest regimen utilization. There was however, general poor utilization of the regimen across the health centers in the LGA.

Table 4: Mectizan supply and utilization in health centres in Ohaozara LGA from 2010 to 2014

\begin{tabular}{|l|c|c|c|}
\hline Health Centers & Number supplied & Number utilized & Utilization\% \\
\hline Obiozara H/C & 478,043 & 13,000 & 2.72 \\
\hline Agugun H/C,Uburu & 478,043 & 36,600 & 7.66 \\
\hline Urban H/C, Okposi & 478,043 & 18,000 & 3.77 \\
\hline Ugwulangwu H/C & 478,043 & 11,430 & 2.39 \\
\hline Ndiunuhu H/C & 478,043 & 20,420 & 4.27 \\
\hline Ojigwe H/C & 478,043 & 13,052 & 2.73 \\
\hline Total & 478,043 & 112,502 & 23.53 \\
\hline
\end{tabular}

The intervention total percentage of utilization was $12.72 \%$, with Oshiri Model Health Centre using $2.95 \%$ of supply $\&$ being the health center with highest drugs utilization while Abaomege Health Centre minimally utilized the supplied regimen was $1.35 \%$. 
Table 5: Mectican supply and utilization in health centres in Onicha LGA from 2010 to 2014

\begin{tabular}{|l|c|c|c|}
\hline Health Centre & Number supplied & Number utilized & Utilization\% \\
\hline Oshiri H/C & 983,970 & 23,552 & 2.39 \\
\hline Isu H/C & 983,970 & 18,900 & 1.92 \\
\hline Abaomege H/C & 983,970 & 13,270 & 1.35 \\
\hline Onicha H/C & 983,970 & 20,200 & 2.05 \\
\hline Oshiri Model H/C & 983,970 & 29,000 & 2.95 \\
\hline Ukawu H/C & 983,970 & 20,200 & 2.05 \\
\hline Total & 983,970 & 125,122 & 12.72 \\
\hline
\end{tabular}

The overall percentage utilization of mectizan in the health centres of Ivo LGA was 23.99\%. Akaeze Model Health Centre had the highest percentage utilization of the intervention while Ekembe Health Centre highly underutilized the regimen.

Table 6: Mectizan supply and utilization in health centres in Ivo LGA from 2010 to 2014

\begin{tabular}{|l|c|c|c|}
\hline Health Centres & Number supplied & Number utilized & Utilization\% \\
\hline Ishiagu Basic H/C & 457,122 & 21,800 & 4.77 \\
\hline Akaeze Model H/C & 457,122 & 22,820 & 4.99 \\
\hline Ihie H/C & 457,122 & 16,051 & 3.53 \\
\hline Iyioji H/C & 457,122 & 20,724 & 4.53 \\
\hline Akaeze Basic H/C & 457,122 & 14,530 & 3.18 \\
\hline Ekembe H/C & 457,122 & 13,650 & 2.99 \\
\hline Total & 457,122 & 109,675 & 23.99 \\
\hline
\end{tabular}

\section{Discussion}

Majority of the inhabitants of the study areas had high knowledge of onchocerciasis and its signs and symptoms. There was also good knowledge of ivermectin as a drug for the treatment of river blindness. Some of the users of the drug explained the side effects. However, this did not agree with Mbaneme et al. (2012), who reported poor knowledge of mode of transmission of onchocerciasis and use of ivermectin in the control of onchocerciasis in their study at Idemili North LGA of Anambra State in Nigeria.

Though there were variations in levels of the supply of the drugs to the various health centres of the Local Government Areas (LGAs), the present results indicated a sufficient delivery of the drugs. The observed differences in levels of the supply to the health centres could be attributed to differences in political interests and strengths of the governments in-charge of the different LGAs; as some of the LGA chairmen are more influential than others, as regards to the acquisition of such treatment regimens to their people. The observed differences in supply could also have resulted from already developed distribution formula, if any, that might be dependent on populations of the inhabitants of the areas.

There was on overall poor utilization of the regimen all over the years, with areas of high drug supply making very low utilization of the regimen. Findings suggested that the poor utilization could have resulted from adverse reactions of the drug, poor attitudes of drug distributors, defaulting from continual yearly compliance to drug utilizations, relocations encouraged by massive migrations from rural to urban areas, proliferation of religious organizations with religious beliefs that forbid taking of drugs, poor sensitization and low level of health education, difficulty of abstaining from alcoholic drinks while taking drugs, poor incentives to the village-based health workers (VBHWs), non-proximity of health centres to the users of the drugs, and communal clashes and conflicts. Earlier reports on similar topics have supported some of the reasons for poor utilization of the regimen. Prozesky et al. (2001) reported that some health teams feel reluctant to supervise or fully support such a programme. The delivery (supply) and utilization of mectizan are two different but complementary actions that must be completed for effective control of onchocerciasis. The prevalence of river blindness could 
still be maintained in a community irrespective of free supply of ivermectin if compliance in utilization of the drug is not maintained. Okoye et al. (2008) reported persistence of high prevalence of onchocerciasis in a re-assessment study carried out in Etteh community after a decade of mass mectizan chemotherapeutic intervention. They attributed the persistent high prevalence of the disease to inefficiency and inconsistency in the distribution of mectizan and suggested further study to investigate details of mectizan distribution in order to find out why prevalence was persistent.

Since primary health care workers have been reported to be the main contact with the community for ivermectin distribution (Burnham and Mebrahtu, 2004), it seems that motivating them morally and financially could be a panacea to the success of the control of the disease, which could be achieved through great compliance with intake of the drug. Wagbatsoma and Okojie (2004) reported that there was a need to improve people's attitude towards the onchocerciasis and improve disease awareness through appropriate health education, which will encourage the acceptance of ivermectin as adequate treatment and compliance to the treatment regimen to reduce morbidity and promote self-esteem.

Sauerbrey (2008) reported that the key factor in onchocerciasis elimination was mass administration of the antiparasitic drug ivermectin (trade name: Mectizan ${ }^{\circledR}$ ) drug. Generally, various control programs were established aimed to stop onchocerciasis from becoming a public health problem. The first was the Onchocerciasis Control Programme (OCP), a joint effort of the WHO, the World Bank, the United Nations Development Programme, and the UN Food and Agriculture Organization was considered to be a success, and came to an end in 2002. Continued monitoring ensures onchocerciasis cannot reinvade the area of the OCP (WHO, 2010). The WHO (2015) declared that the first countries to receive verification of elimination were Colombia in 2013, Ecua dor in 2014, and Mexico in 2015, and Guatemala submitted a request for verification.

On the other hand, in Sudan that boarded Egypt, Abdel Fattah et al. (1985a, b) reported the prevalence of onchocerciasis volvulus. Besides, one species of Simuliidae was reported in Egypt (Steyskal and el-Bahy, 1967). CDC (2011) upon request from Sudan Ministry of Health to assist in studying the increase and geographic clustering of an illness resulted in head nodding and seizures, with suspected outbreak emergence. The condition predominantly affected children aged 5-15 years in South Sudan from the states of Western and Central Equatoria and in Northern Uganda and southern Tanzania. The CDC team carried out a casecontrol study. They reported that 38 matched case-control pairs were enrolled from two different communities: Maridi and Witto. They added that onchocerciasis was more prevalent among case-patients, whether infection preceded or followed nodding syndrome, but its onset was unknown. Priorities for nodding syndrome investigations include improving surveillance to monitor the number of cases and their geographic distribution and continued work to determine the etiology of the syndrome. In Egypt, El-Massry and Derbala (2000) examined 3376 imported and 200 local camels between September 1997 and August, 1998 for natural infection with $O$. fasciata nodules and subsequently for adult parasites. They reported that imported camels had the higher infection rate $(2.75 \%)$, while those of local origin showed no palpable or detected Onchocerca nodules. Zarroug et al. (2014) stated that Abu Hamed, the northernmost onchocerciasis focus in the world, is located along the River Nile banks in the Nubian Desert. Hydroelectric dams can alter activity of black flies and may provide breeding sites for black fly. Merowe Dam, the largest hydropower project in Africa, was built west of Abu Hamed focus in 2009. They concluded that continuous entomological surveys are needed to 
monitor presence of black fly vectors and its impact on the disease spreading.

In Kuwait, Abdul-Salam and Al-Taqui (1995) reported that 32 (11\%) camels (Camelus dromedarius) were infected with Onchocerca-like microfilaria, and that the highest monthly incidence $(27 \%)$ was detected in June at the end of spring. They concluded that harsh environmental conditions in $\mathrm{Ku}-$ wait during summer either arrest development of microfilariae or influence their diurnal distribution in tissues of the camel.

In the Kingdom of Saudi Arabia, Helmy and Al Mathal (2003) in Asir Region diagnosed three patients locally acquired chronic hyperactive form of onchocerciasis volvulus. They were referred for ivermectin treatment by the clinicians in the Specialized Hospital.

Generally speaking, ivermectin was used to control the ecto- and endoparasites (mites and mainly nematodes) of livestock and pets (Morsy et al, 2001a), and proved effective against zoonotic Sarcoptes scabiei infesting human (Morsy et al, 2001b). Besides, it was also used as anti-filarial chemotherapy in man since it killed the immature worms (microfilariae) that cause the harmful symptoms of onchocerciasis (Omura, 2008). After ivermectin administration to mammals, its chemical decomposition by metabolism was generally low (McKellar and Gokbulut, 2012), and between $62 \%$ and $98 \%$ of the ivermectin inoculated might be excreted as unchanged residue in the feces (Kryger et al, 2005).

Richard-Lenoble et al. (2001) in Yemen identified as microfilaria type Onchocerca but it did not belong to species volvulus, or to species ochengi, which seemed to be the clinical picture of sowda resulted from the developing onchocerciasis of animal origin and not identified as to day. They concluded that the ivermectin, therapeutic of choice for African onchocerciasis in the annual unique cure seemed less effective in the coverage of sowda, and that case rehearsal of cures every 3 months would be necessary for mass campaigns to limit the transmission. Eberhard et al. (2012) reported that there were limited pharmacological choices for management of the ocular onchocerciasis. Ivermectin is the recommended treatment for microfilaria but has no effect on the adult worms; Dana et al. (2015) in southwestern Ethiopia recommended that the onchocerciasis endemicity level was the mesoendemic. They added that thus the intervention using ivermectin treatment should be implemented to reduce the burden of onchocerciasis.

\section{Conclusion}

The community-based distributors should be able to mobilize and educate the populace, dispense the drugs, maintain records, monitor and treat adverse drug reactions. There were sufficient supplies of mectizan to the communities of study area but there were high levels of under utilizations of the regimen. Intensification of efforts to achieve improved utilization of mectizan is advocated for effective control of the river blindness. Ivermectin $\left(=\right.$ Mectizan $\left.^{\circledR}\right)$ proved to be effective not only in onchocerciasis but also in other ecto- and endo-parasites.

No doubt, the climatic changes resulted from the dams have a positive impact on the onchocerciasis and black fly vector spreading to other regional countries. Consequently, regional collaboration is a must to improve the vector control, diagnostic methods and to support ongoing efforts for the elimination the onchocerciasis, which causes the multiple skin manifestations, as well as the river blindness.

\section{References}

Abdel Fattah, A, Youssif, NM, Shenouda, OA, Morsy, TA, 1985a: On the treatment of onchocerciasis in the Sudan. J. Egypt. Soc. Parasitol. 15, 1:329-33.

Abdel Fattah, A, Youssif, NM, Shenouda, OA, Morsy, TA, 1985b: On the diagnosis of onchocerciasis in the Sudan. J. Egypt. Soc. Parasitol. 15, 2:365-70.

Abdul-Salam, J, Al-Taqui, M, 1995: Seasonal prevalence of Onchocerca-like microfilaria in camels in Kuwait. J. Egypt. Soc. Parasitol. 25, 1: 19-24.

Brattig, NW, 2004: Pathogenesis and host responses in human onchocerciasis: impact of $\mathrm{On}$ - 
chocerca filariae and Wolbachia endobacteria. Microbes Infect. 6:113-28.

Burnham, G, Mebrahtu, T, 2004: Review: The delivery of ivermectin $\left(\right.$ Mectizan $\left.^{\circledR}\right)$. Trop. Med. Int. Hlth. 9, 4:26-44.

CDC, 2011: Nodding syndrome - South Sudan, 2011. MMWR Morb Mortal Wkly Rep. 61, 3: $52-4$.

Cox, FEG, 1993: Modern Parasitology: A Textbook of Parasitology, $2^{\text {nd }}$ Edition. ISBN: 978-0632-02585-5.

Crump, A, Morel, CM, Omura, S, 2012: The onchocerciasis chronicle: from the beginning to the end? Trends Parasitol. 28, 7:280-7.

Dana, D, Debalke, S, Mekonnen, Z, Kassahun, W, Suleman, S, et al, 2015: A communitybased cross-sectional study of the epidemiology of onchocerciasis in unmapped villages for community directed treatment with ivermectin in Jimma Zone, southwestern Ethiopia. BMC Publ. Hlth. Jul 1;15:595.doi:10.1186/s12889-015-1888 $-\mathrm{X}$.

Eberhard, ML, Sims, AC, Bishop, HS, Mathison, BA, Hoffman, RSI, 2012: Ocular zoonotic onchocerca infection in a resident of oregon, Amer. J. Trop. Med. Hyg. 87, 6: 1073-5.

El-Massry, AA, Derbala, AA, 2000: Evidence of Onchocerca fasciata (Filarioidea: Onchocercidae) in camels (Camelus dromedarius): I-prevalence, nodular lesions appearance and parasite morphology. Vet. Parasitol. 88, 3/4:305-12.

Helmy, MM, Al Mathal, IM, 2003: Human infection with Onchocerca volvulus in Asir District (Saudi Arabia). J. Egypt. Soc. Parasitol. 33, 2:385-90.

Kryger, U, Deschodt, C, Scholtz, CH, 2005: Effects of fluazuron and ivermectin treatment of cattle on the structure of dung beetle communities. Agric. Ecosys. Environ. 105:649-56.

Mbaneme, FCN, Onuzulike, N, Mbaneme, E O, 2012: Effect and management of onchocerciasis among parents in the lower Benue Trough of Nigeria. J. Environ. Earth Sci. 2, 11:50-9.

McKellar, QA, Gokbulut, C, 2012: Pharmacokinetic features of the antiparasitic macrocyclic lactones. Curr. Pharm. Biotechnol. 13, 888-911.

Morsy, TA, Abdel-Mawla, MY, Morsy, ATA, 2001a: The effect of ivomec super on dogs experimentally infested with Sarcoptes scabiei isolated from a patient. J. Egypt. Soc. Parasitol. 31, 1:79-85.
Morsy, TA, Habib, KS, Haridy, FM, 2001b: Ivermectin and clorsulon (ivomec super) in the treatment of goats naturally infested with scab mites and biting lice. J. Egypt. Soc. Parasitol. 31, 2:373-9.

Okoye, IC, Ubachukwu, PO, Okeke, V, Obiezue, RN, Onyishi, GC, 2008: Reassessment of onchocerciasis prevalence in Etteh after a decade of mass mectizan chemotherapeutic intervention: Preliminary report. Anim. Res. Int. 5, 1:827-30.

Omura, S, 2008: Ivermectin: 25 years and still going strong. Int. J. Antimicrob. Agents 31, 2: 91-8.

Prozesky, D, Blitz, J, Gibson, R, 2001: An investigation into the administration requirements of the African Programme for Onchocerciasis, Faculty of Health Sciences, Pretoria.

Rea, PA, Zhang, V, Baras, YS, 2010: Ivermectin and River Blindness. Am. Scientist 98, 4: 294-303.

Richards, FO, Jr, Boatin, B, Sauerbrey, M, Seketeli, A, 2001: Control of onchocerciasis today: Status and challenges. Trends Parasitol. 17, 12:558-63.

Richard-Lenoble, D, al Qubati, Y, Toe, L, Pisella, PJ, Gaxotte, P, et al, 2001: Human onchocerciasis and "sowda" in the Republic of Yemen. Bull. Acad. Natl. Med. 185, 8:1447-61.

Sauerbrey, M, 2008: The Onchocerciasis Elimination Program for the Americas (OEPA). Ann. Trop. Med. Parasitol. 102 Suppl 1: 25-9.

Steyskal, GC, El-Bahy, WS, 1967: A List of Egyptian Diptera with a Bibliography and Key to Genera. The Egyptian Ministry of Agriculture; Tech. Bull. No. 3.

Wagbatsoma, VA, Okojie, OH, 2004: Psychosocial effects of river blindness in a rural community in Nigeria. J. R. Soc. Promot. Hlth. 124, 3134-6.

WHO, 2010: Integrated preventive chemotherapy for neglected tropical diseases: estimation of the number of interventions required and delivered 2008-2009.

WHO, 2012: Integrated preventive chemotherapy for neglected tropical diseases: estimation of the number of interventions required and delivered 2009-2010, Geneva.

WHO, 2015: Onchocerciasis. Retrieved3 October 2015, Geneva. 